\title{
Les enjeux territoriaux du TCSP de la Martinique
}

Territorial stakes of «TCSP » of Martinique

\section{Colette Ranély Vergé-Dépré}

\section{Q OpenEdition}

\section{Journals}

\section{Édition électronique}

URL : http://journals.openedition.org/rge/3515

DOI : $10.4000 /$ rge. 3515

ISSN : 2108-6478

\section{Éditeur}

Association des géographes de l'Est

\section{Édition imprimée}

Date de publication : 15 juin 2012

ISSN : 0035-3213

\section{Référence électronique}

Colette Ranély Vergé-Dépré, "Les enjeux territoriaux du TCSP de la Martinique », Revue Géographique de l'Est [En ligne], vol. 52 / 1-2 | 2012, mis en ligne le 16 octobre 2012, consulté le 10 décembre 2020. URL : http://journals.openedition.org/rge/3515 ; DOI : https://doi.org/10.4000/rge.3515

Ce document a été généré automatiquement le 10 décembre 2020.

Tous droits réservés 


\title{
Les enjeux territoriaux du TCSP de la Martinique
}

\author{
Territorial stakes of «TCSP » of Martinique
}

Colette Ranély Vergé-Dépré

1 Petite île française des Antilles, la Martinique $\left(1128 \mathrm{~km}^{2}\right)$ est confrontée à la saturation des axes routiers desservant l'agglomération foyalaise, le principal pôle humain et économique de l'île. Fort-de-France est au cœur d'un réseau routier en étoile et est un point de passage obligé entre quasiment toutes les parties du territoire martiniquais. Avec en moyenne une voiture particulière pour deux habitants (205524 voitures pour 402000 Martiniquais au 01/01/2010 d'après l'INSEE), le trafic routier ne cesse de progresser alors que les carences des transports collectifs ne permettent pas de proposer une offre alternative attractive pour les populations. Après des décennies d'immobilisme, tant de la part de l'État, des autorités organisatrices de transport (AOT) ou des corporations de transporteurs, les collectivités territoriales ont initié, à la fin des années 1990, une réflexion sur la modernisation des transports publics par la mise enplace d'un Transport en Commun en Site Propre (TCSP) au sein de l'agglomération foyalaise, avec une extension aux communes voisines envisagée à plus long terme. Un projet ambitieux (deux lignes de tramway sur pneu entre Fort-de-France et Le Lamentin) a été adopté en 2003 et devait être opérationnel en 2011. Mais l'ampleur des investissements, les aléas politiques, les difficultés techniques, sont à l'origine des retards dans la réalisation du TCSP. Ces difficultés sont régulièrement relayées dans les médias qui n'hésitent pas à qualifier le TCSP de « serpent de mer $»^{1}$ ou « d'arlésienne »: on en parle beaucoup, mais on ne le voit toujours pas...

2 Reporté à l'horizon 2015, le projet doit sans doute subir des modifications, notamment dans le choix du matériel roulant. Dans ce contexte, le TCSP de la Martinique a-t-il les moyens de répondre aux attentes des élus, des aménageurs et des populations? Plus qu'une solution technique visant à décongestionner les axes les plus fréquentés de l'île, le TCSP est l'occasion de repenser une politique de développement, associant transports et mobilités, mais aussi urbanisme, aménagement des territoires, bien-être social, activités économiques et environnement. Autant de défis à relever pour un 
projet qui, pour l'heure, a bien du mal à se concrétiser et qui est l'un des rares exemples d'aménagement de ce type en outre-mer².

\section{Territoires et acteurs face au projet du TCSP de la Martinique}

3 Ce cas illustre les enjeux existant autour de la prise de décision et la mise en œuvre d'un projet qui a dû être adapté aux difficultés rencontrées, quelles soient d'ordre technique ou politique. Situé à la croisée de plusieurs domaines d'intervention qui peuvent se révéler conflictuels, ce type d'aménagement est l'exemple même d'un projet urbain « négocié » [Hamman (dir.), 2011].

\section{A. Le contexte et les objectifs du projet}

4 Le poids centralisateur de l'agglomération foyalaise n'a cessé de se renforcer au cours des dernières décennies. Cumulant les fonctions de capitale politique et économique, Fort-de-France est la commune la plus peuplée de l'île et a étendu son influence sur les communes voisines, sans qu'il soit véritablement facile d'en tracer les limites spatiales exactes (figure 1).

Figure 1. L'agglomération de Fort-de-France

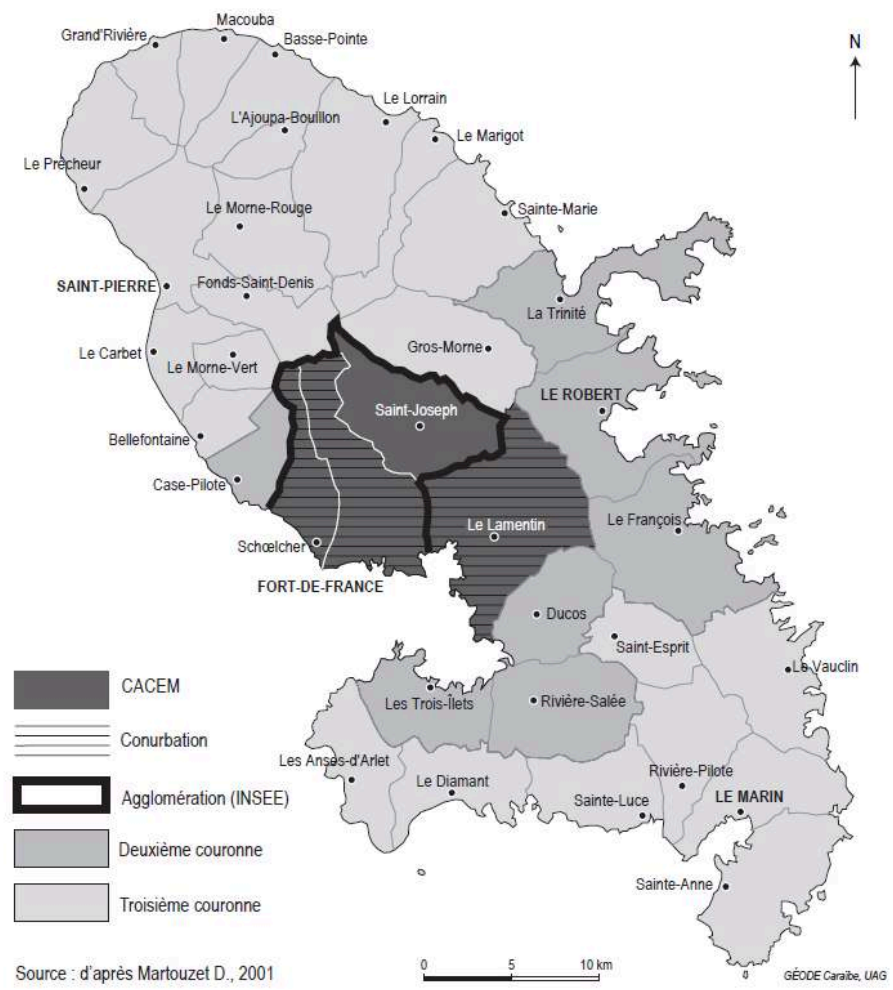

5 Selon les critères de l'INSEE, l'agglomération comprend trois communes : Fort-deFrance (89 000 habitants en 2008), Schœlcher (21 737 habitants) et Saint-Joseph (16 966 habitants), pour un total de 127703 habitants. Cette entité ne correspond pourtant pas à la réalité économique et à l'espace vécu des populations car elle n'inclut pas la commune du Lamentin (39 410 habitants) qui concentre de nombreux équipements 
(aéroport international, zones industrielles et commerciales) et qui est reliée à Fort-deFrance par des flux de circulation denses. La conurbation foyalaise est plus proche de la réalité des déplacements puisqu'elle correspond aux communes de Fort-de-France, Le Lamentin et Schœlcher. Créée en 2000, la Communauté d'Agglomération du Centre de la Martinique (CACEM) regroupe quant à elle les quatre communes (Fort-de-France, Le Lamentin, Schœlcher et Saint-Joseph) et correspond plus généralement au périmètre désigné par "agglomération foyalaise». Ainsi définie, celle-ci concentre $42 \%$ de la population (167 113 habitants en 2008), $60 \%$ des emplois, sur seulement $15 \%\left(171 \mathrm{~km}^{2}\right)$ du territoire martiniquais.

6 Les tendances récentes de la croissance de l'agglomération ont montré que l'habitat a suivi une direction privilégiée orientée du sud vers le nord (du littoral vers les pentes au nord), alors que les activités économiques se sont implantées le long d'un axe estouest, reliant les villes du Lamentin, de Fort-de-France et de Schœlcher qui constitue ainsi un axe majeur de communication. Les trafics atteints (jusqu'à 110000 véhicules par jour sur l'autoroute A1) sont du même niveau que ceux recensés aux entrées des grandes métropoles françaises : à Toulouse par exemple, on recense sur le périphérique des trafics journaliers équivalents, mais pour une agglomération cinq fois plus peuplée (DREAL Midi-Pyrénées, 2012). Les phénomènes de périurbanisation et de mitage urbain contribuent à étendre et à densifier l'espace urbanisé, notamment en direction du sudcaraïbe et du centre-atlantique. Malgré des travaux d'agrandissement du réseau routier, le déséquilibre entre la capacité des voiries et les flux qu'elles supportent conduit à une saturation des principaux axes.

7 Le choix de la réalisation d'un TCSP, d'abord entre Fort-de-France et Le Lamentin, vise à améliorer les déplacements des populations et à leur proposer une offre de transport public plus attractive et efficace, utilisant une voie spécialement réservée et protégée de la circulation automobile (en site propre). Il permet « l'articulation entre l'offre en transports collectifs du réseau communautaire (CACEM) et les transports interurbains irrigant une grande partie de la Martinique vers l'agglomération centrale » (SMTCSP, SYSTRA, 2006, vol.2, p.17). Il souhaite également promouvoir le transport multimodal puisque son tracé dessert l'aéroport international, la gare multimodale de la Pointe Simon et la gare maritime inter-îles à Fort-de-France. Enfin, le TCSP peut améliorer l'accessibilité au centre-ville, permet de mettre en œuvre un partage de la voirie, réduisant ainsi les risques d'insécurité et les temps de parcours, et contribue à réduire les nuisances environnementales générées par les déplacements en véhicules à moteur.

\section{B. Un jeu d'acteurs complexe}

8 Face à la nécessité de remédier au problème de déplacement des personnes dans l'agglomération foyalaise, les différentes AOT ont chacune intégré la réflexion sur la mise en place d'un TCSP dans les grandes orientations relevant de leurs domaines de compétences propres. Les initiatives, d'abord individuelles, sont devenues, par nécessité, plus collectives malgré les difficultés pour mettre en cohérence des actions visant à élaborer et à concrétiser le projet de TCSP. Espace insulaire restreint, la Martinique est à la fois Département et Région, et l'un des principaux obstacles au projet est la multiplicité des acteurs institutionnels se partageant les compétences en matière de transports sur des périmètres emboîtés. La juxtaposition et la superposition des compétences sur les territoires institutionnels, héritées de la Loi d'orientation sur les transports intérieurs (1982), ont donné lieu comme ailleurs à un nécessaire 
«bricolage institutionnel»(Bavoux et al., 2005, p.144) dont l'efficacité n'est pas toujours pleinement réalisée.

9 Le Conseil Général, autorité organisatrice des transports non urbains de personnes a initié, dans la seconde moitié des années 1990, des débats sur la modernisation des transports publics avec les syndicats des transporteurs, le grand public, les acteurs économiques, et a mené diverses études sur la mise en place d'un TCSP. Le Conseil Régional, dans son Schéma d'Aménagement Régional (1998), a cherché à adapter la trame routière principale de l'île aux nécessités de développement de l'armature urbaine. La Commission européenne, sollicitée par le Conseil Général pour obtenir une aide financière pour son projet de TCSP, a accepté de le soutenir dans le cadre des Grands projets européens, mais a exigé d'avoir un interlocuteur unique réunissant toutes les collectivités concernées : le Département, la Région, les villes de Fort-deFrance, Le Lamentin et Schœlcher. Le 14 décembre 2000 a ainsi été créé le Syndicat Mixte du Transport Collectif en Site Propre (SMTCSP) de la Martinique qui a été chargé de la maîtrise d'ouvrage du projet. Il réunit le Département, la Région et la CACEM, ellemême mise en place fin décembre 2000. Au sein de son Périmètre des transports urbains (PTU), la CACEM a adopté, en décembre 2003, un Plan de déplacement urbain (PDU) qui prévoit notamment la réalisation d'un site propre principal, de direction estouest, entre Le Lamentin, Fort-de-France et Schœlcher, et envisage des sites propres complémentaires d'agglomération, sur des axes routiers très fréquentés et de direction méridienne.

L'absence d'une AOT unique, dans un contexte de clivages politiques récurrents ${ }^{3}$, a souvent ralenti la prise de décision et la réalisation d'un projet complexe tant au niveau financier que technique et urbanistique. La loi Grenelle de l'environnement $\mathrm{n}^{\circ} 2$, promulguée le 12 juillet 2010, stipule pourtant qu'«il peut être désigné, dans les départements et régions d'outre-mer, une autorité organisatrice de transports unique, et défini un périmètre unique de transports qui se substitue à tous les périmètres de transports urbains existants et couvre l'ensemble du territoire de ces collectivités » (art.52, abrogé par l'ordonnance $n^{\circ} 2010-1307$ du 28 octobre 2010, article 7). Pour l'heure, cela n'a pas pu être mis en place alors que le SMTCSP ne dispose pas des moyens pour assurer efficacement sa mission. Il a dû, par exemple, longuement négocier avec la CACEM pour que celle-ci lui cède ses compétences sur le TCSP mais il ne peut intervenir sur les autres réseaux de la CACEM et assurer ainsi une coordination des transports. De plus, n'ayant ni ressources propres ni compétence de gestion, le syndicat mixte se heurte à des difficultés financières; c'est notamment le cas pour l'acquisition de parcelles sur le tracé du TCSP, opération en cours depuis plus de dix ans et pour laquelle il dépend des financements votés par la Région. Le projet a été inscrit, dès l'année 2000, au Document unique de programmation (DOCUP) 2000-2006, puis au Programme Opérationnel (PO) FEDER de 2007-2013, afin de bénéficier des fonds structurels européens. Le budget de l'opération a été évalué à l'origine à 245,81 millions d'euros, financé à près de $60 \%$ par les collectivités locales (Conseil Régional : 124,86 M€. Conseil général : 15,2 M€. CACEM : 4,01 M€ et SMTCSP : 2,25 M€), $36 \%$ par le FEDER $(88,49 \mathrm{M} €)$ et $4 \%$ par l'État $(11 \mathrm{M} €)$. Les récents changements politiques opérés depuis les élections régionales (2010) et cantonales (2011) permettent d'atténuer les divergences entre les deux assemblées qui sont désormais dirigées par une même coalition, "Ensemble, pour une Martinique nouvelle», créée à l'initiative de Serge 
Letchimy, chef du Parti Progressiste Martiniquais et élu Président du Conseil Régional en 2010.

\section{Les vicissitudes du projet et son évolution}

11 Après plusieurs études et débats portant, par exemple, sur le choix du tracé et du matériel roulant, le SMTCSP de la Martinique a retenu, en 2003, un projet adopté par le Conseil Général et le Conseil Régional en séance plénière conjointe. Plus qu'un simple projet d'infrastructure, celui-ci ambitionnait d'apporter une réponse au problème de déplacement à l'échelle de l'agglomération mais aussi des communes sous l'influence de la conurbation foyalaise. Dans un premier temps - à l'horizon 2011, il était prévu la construction d'un axe est-ouest reliant Fort-de-France au Lamentin et qui devait remplacer toute autre liaison interurbaine de transport collectif (figure 2). Il serait ensuite prolongé, à plus long terme, vers le nord (Schœlcher), l'est (Le Robert) et le sud (Ducos/Rivière Salée).

Figure 2. Le TCSP de la Martinique, un projet à long terme

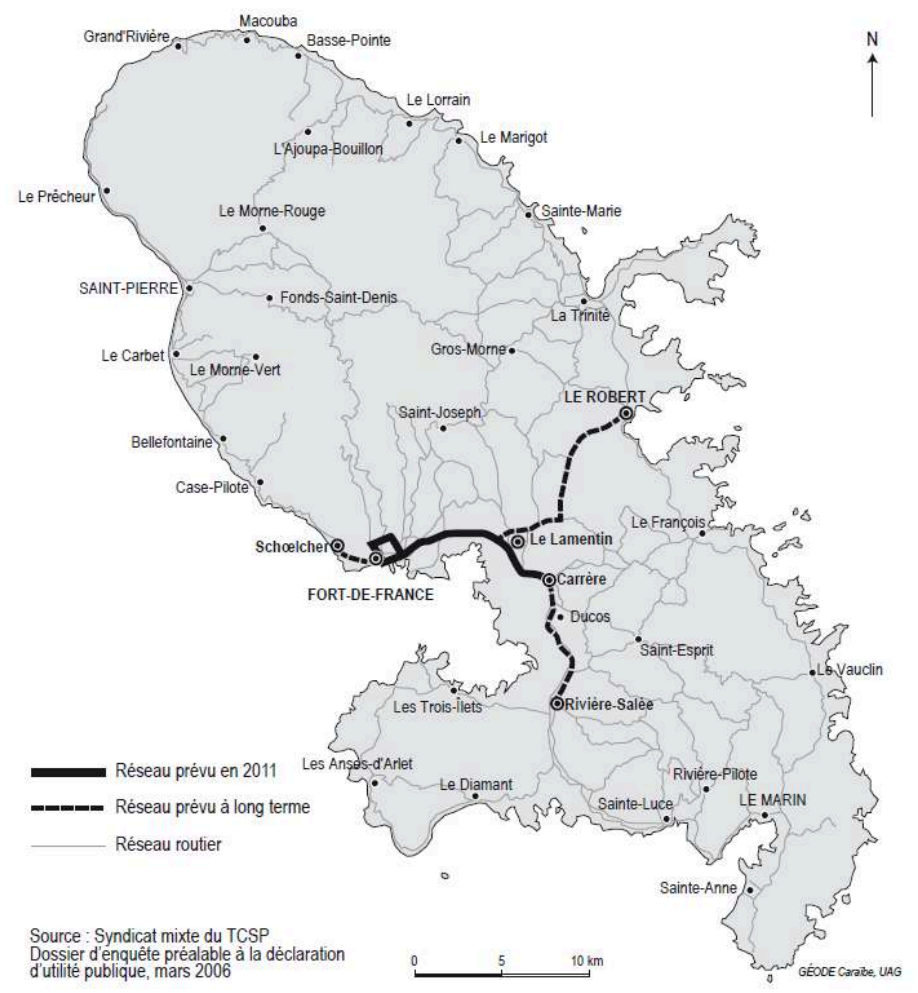

Selon la première phase du projet, le tracé du TCSP (d'une longueur totale de 13,9 km et équipé de 18 stations) dessert le centre-ville de Fort-de-France et se dédouble vers Le Lamentin: une branche se dirige vers la place Mahault, à proximité de la RN1, une autre conduit à l'aéroport et se termine à Carrère (figure 3). Les terminus sont conçus comme des pôles d'échanges accueillant les liaisons d'autobus en provenance du reste de l'île et seront dotés de parcs-relais où les automobilistes pourront laisser leurs véhicules avant d'emprunter le TCSP. Le projet souhaite donc renforcer l'intermodalité, celle-ci trouvant sa pleine réalisation à la gare multimodale de la Pointe Simon, à Fort- 
de-France, où aux transports terrestres s'ajoutent les dessertes maritimes vers les Trois-Îlets, de l'autre côté de la baie.

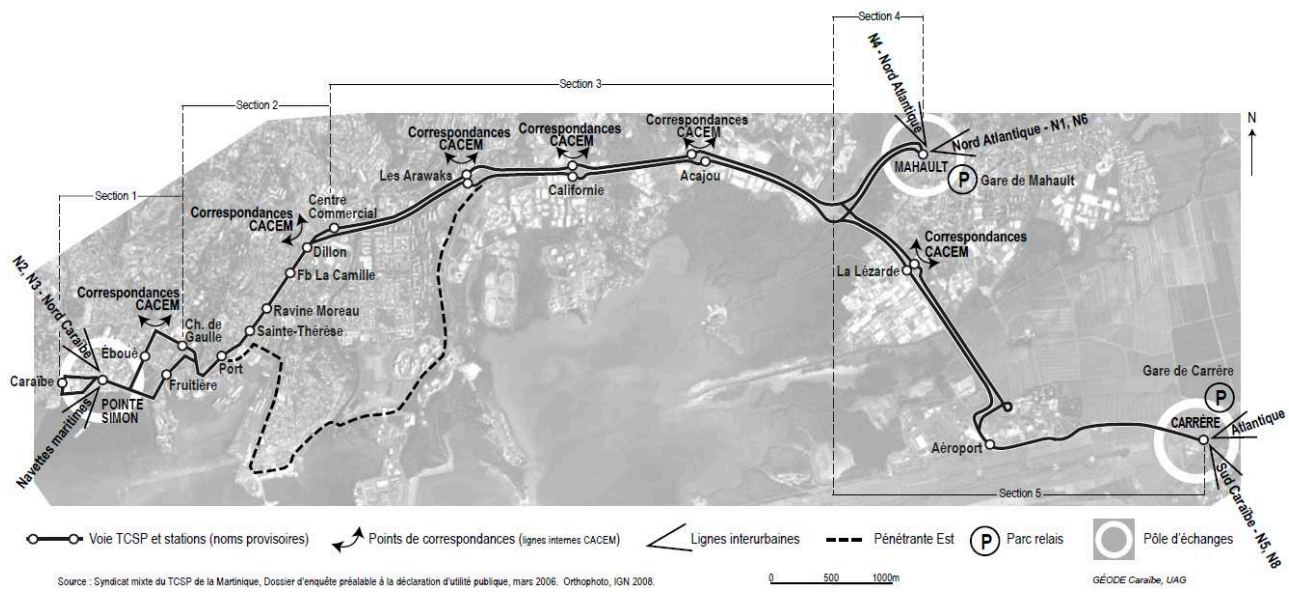

Figure 3. Le TCSP de la Martinique, un projet structurant

Le tracé du TCSP emprunte celui des axes routiers les plus fréquentés, le choix de l'autoroute soutenu par le Conseil Régional, principal pourvoyeur de fonds, l'ayant finalement emporté. Il a été divisé en cinq segments, dont les deux premiers sont situés en zones très urbanisées. La réalisation des travaux a été répartie entre les trois membres du SMTCSP. Le Conseil Général est maître d'ouvrage dans la section du centre-ville foyalais (section 1). Le Conseil Régional, responsable de la gestion de l'autoroute et des routes nationales, est maitre d'ouvrage dans les sections restantes et doit financer le matériel roulant et l'atelier-dépôt de Carrère. La CACEM, autorité organisatrice des transports urbains à l'intérieur de son PTU, est responsable de l'aménagement des gares terminus de la Place Mahault et de Carrère. L'aménagement d'une voie routière dénommée "Pénétrante Est » dans le projet, est indissociable du TCSP car elle est nécessaire à la réalisation des travaux de la section 2 (avenue Maurice Bishop) : elle sert de déviation du trafic entrant à Fort-de-France, en contournant, par le sud, les quartiers de Dillon et de Volga, en empruntant l'actuelle voie portuaire.

Parmi les différents systèmes proposés (Région, SETEC, 2001), le SMTCSP avait retenu, en juillet 2003, le tramway sur pneu "flexible» pour des raisons de coûts d'investissement et d'exploitation, de performances techniques et de capacité de transport : 140 personnes par véhicule, ce qui permettrait de transporter environ 3000 passagers par sens en heure de pointe, à raison d'un départ des gares toutes les 6 minutes (SMTCSP, SYSTRA, 2006). Le trajet entre les terminus de Carrère et la Pointe Simon devait s'effectuer en une vingtaine de minutes. À l'instar de ce qui a déjà été réalisé depuis le renouveau du tramway dans les métropoles de la France hexagonale ${ }^{5}$, y compris celles de taille moyenne soit environ 200000 habitants (Angers, Le Mans ou Caen), c'est donc un mode de transport innovant qui aurait été proposé aux usagers afin de les convaincre de modifier leurs habitudes de déplacements et d'assurer une plus grande cohésion entre les différentes composantes du territoire martiniquais. Le monorail, défendu par un "Comité pour la modernisation du transport public en Martinique par la promotion du monorail ", lui-même soutenu par une union syndicale professionnelle de transporteurs, a été écarté pour son coût jugé excessif. 
Depuis son lancement, le projet a fait l'objet d'une concertation avec différents acteurs politiques, économiques, sociaux, associatifs et a bénéficié de campagnes d'information du grand public. La lassitude des populations face aux difficultés de déplacement explique que les Martiniquais adhèrent globalement à ce projet, alors que certaines professions (conducteurs de taxis et de taxis collectifs, concessionnaires et assureurs de véhicules automobiles, etc.) se sont montrées plutôt réticentes. L'ampleur et la complexité des opérations à réaliser, des difficultés financières et des dissensions politiques, ont notamment retardé la réalisation du TCSP : alors que celui-ci devait être opérationnel en 2011, force est de constater que le chantier est loin d'être terminé. Pour l'heure, outre la réalisation de diverses études, ont été effectués les travaux d'élargissement de l'autoroute, de la RN1, l'aménagement de l'échangeur du canal du Lamentin et des voies formant une boucle au centre-ville; ce site propre partiel a été ouvert aux transports collectifs existants, permettant ainsi une légère réduction des temps de parcours. Le Conseil Général, qui a également construit la gare de la Pointe Simon, a annoncé au SMTCSP, en janvier 2011, son renoncement à effectuer les travaux concernant la boucle de retournement en centre-ville pour des raisons techniques et financières ${ }^{6}$. Les porteurs du projet ont donc dû l'adapter aux difficultés rencontrées. L'un des points encore actuellement en débat porte sur le franchissement des échangeurs de l'autoroute ${ }^{7}$. Alors que le projet de l'ancienne majorité au Conseil Régional envisageait le passage du TCSP au niveau de la circulation, avec priorité pour celui-ci grâce à des feux tricolores, la nouvelle majorité vient d'initier de nouvelles études de trafics afin d'envisager d'autres solutions techniques moins perturbantes pour la circulation automobile, mais plus coûteuses (construction d'ouvrages dénivelés pour séparer le TCSP des flux automobiles). La mise en service du TCSP a été reportée à l'horizon 2015 et il semble que l'on s'oriente de plus en plus vers le choix d'un bus à haut niveau de service (BHNS) dont la mise en œuvre et l'exploitation sont moins onéreuses. C'est à ce projet que l'État a annoncé, en février 2011, une aide de 11,750 millions d'euros. Sous peine de perdre les crédits européens pour cause du non respect des engagements sur les délais d'exécution, le SMTCSP est dans l'obligation de respecter la nouvelle échéance retenue. La mise en place, en 2014, d'une collectivité unique à la Martinique ${ }^{8}$ réunissant les compétences dévolues au Département et à la Région, devrait peut-être favoriser la finalisation de ce projet qui a longtemps été présenté comme un élément de réponse aux nombreux défis que rencontre la Martinique et ce, dans une perspective de développement durable.

\section{Les enjeux du TCSP sur les systèmes de transport et les mobilités spatiales}

16 Les mobilités spatiales peuvent se définir comme l'ensemble des déplacements des acteurs dans l'espace, quels que soient la durée et la distance du déplacement, les moyens utilisés, leurs causes et leurs conséquences. Selon J.-P. Lévy et F. Dureau (2002, p.6-7), elles dépendent de plusieurs facteurs : «l'accessibilité, c'est-à-dire l'offre qui permet aux individus et aux ménages de réaliser leur mobilité. (...) Le comportement, qui insiste sur le caractère distinctif et culturel de la mobilité. (...) Les contraintes économiques et temporelles qui rendent les mobilités socialement et économiquement sélectives ». 


\section{A. Vers une amélioration de l'offre de transport et de l'accessibilité ?}

Au premier janvier 2010, la Martinique comptait environ $2400 \mathrm{~km}$ de routes, dont $7 \mathrm{~km}$ d'autoroute, $275 \mathrm{~km}$ de routes nationales, $630 \mathrm{~km}$ de routes départementales et 1494 $\mathrm{km}$ de voies communales (figure 4). Le réseau est de type radial, au départ de Fort-deFrance ou du Lamentin, villes reliées par une véritable épine dorsale que constitue la rocade (D41), prolongée par l'autoroute A1. La forte anthropisation du littoral, le caractère montagneux du centre de l'île, la polarisation des activités sur les principaux centres urbains, font que certaines portions du territoire sont encore difficilement accessibles (notamment dans le nord) malgré les améliorations apportées au réseau.

Figure 4. Le réseau routier simplifié de la Martinique

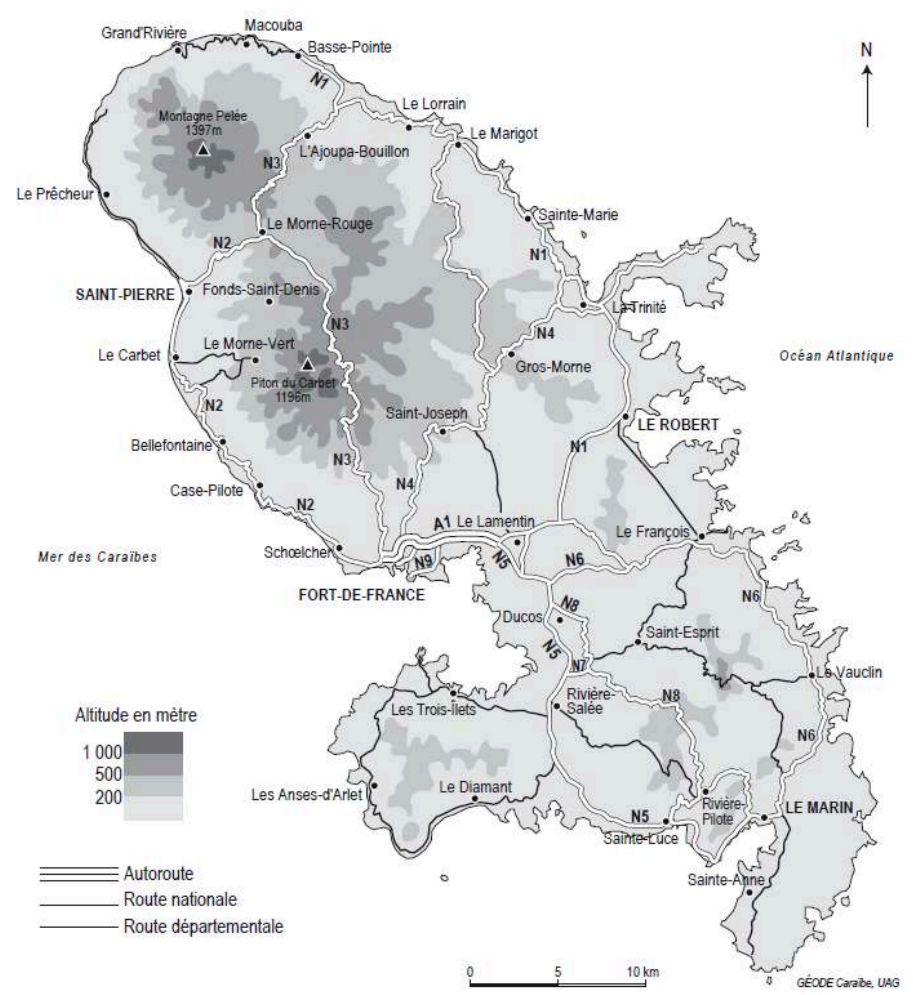

Avec une augmentation moyenne de $4 \%$ par an, le trafic routier se caractérise par une décroissance des flux de l'agglomération foyalaise vers les communes périphériques (figure 5). Les évolutions récentes montrent une forte hausse des trafics en direction du sud et du nord-caraibe, ainsi que vers le nord-atlantique, provoquant ainsi une saturation des axes concernés (N5, N2, N1). Mais le niveau de congestion le plus critique se situe sur l'autoroute (fréquentation journalière de plus de 110000 véhicules). Ce phénomène de saturation du réseau routier ne se limite plus désormais aux heures de pointe et s'est généralisé dans le temps et dans l'espace en raison de l'augmentation du parc automobile (on enregistre, en moyenne, 13000 immatriculations de voitures particulières par an) et de la complexité croissante des mobilités. 
Figure 5. Le trafic automobile à la Martinique en 2010

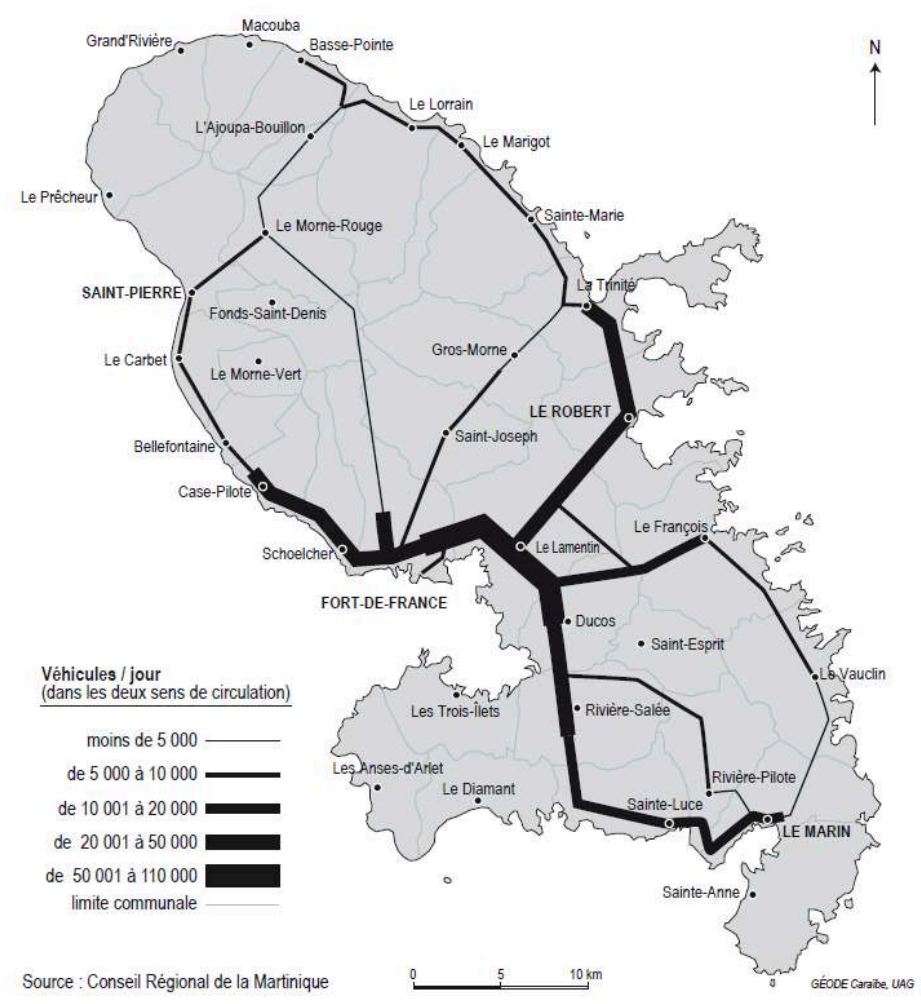

19 Les déplacements sont motivés par de multiples facteurs (travail, études, affaires personnelles, loisirs). En Martinique, la principale cause de mobilité est constituée par les navettes domicile-travail. Selon l'INSEE (RP 2006), $55 \%$ des actifs martiniquais travaillent hors de leur commune de résidence et $79 \%$ des actifs utilisent leur voiture pour se rendre sur leur lieu de travail. Cela s'explique par les atouts que procure l'automobile, symbole de liberté et de réussite sociale, et par le manque d'attraction des services de transports en commun en termes de réseaux, de fréquences, de tarifs, voire de sécurité. La mise en place du TCSP est l'occasion d'entreprendre une restructuration des lignes de bus départementales par un système de rabattement aux terminus de cette infrastructure. Cette réorganisation est déjà engagée au sein des communes de la CACEM afin d'assurer une plus grande cohérence et complémentarité entre le réseau de bus urbains de l'agglomération et le TCSP. En 2010, la CACEM dispose d'un réseau de 49 lignes de bus (dont une trentaine intra-urbaines), régi depuis 2006 par une société d'économie mixte, Mozaïk, délégataire de service public. Les enjeux sont nombreux: permettre une meilleure desserte des territoires, offrir aux populations de toutes les communes de l'île une plus grande égalité des chances de se déplacer en tout lieu, gain de temps lors des déplacements, promotion de la multimodalité en facilitant l'usage successif d'un autocar interurbain ou d'un " taxico » (taxi collectif privé), d'une navette maritime et du TCSP. D'après les hypothèses retenues, le TSCP pourrait transporter 55750 passagers par jour en 2015, soit 14,6 millions de passagers annuels. $60 \%$ d'entre eux seraient d'anciens usagers des transports collectifs, $37 \%$ seraient d'anciens utilisateurs de la voiture et $6 \%$ seraient des passagers induits par les gains de mobilité (SMTCSP, SYSTRA, 2006, p.129). 


\section{B. Les enjeux pour les politiques publiques : quelles régulations des mobilités par l'action publique?}

Le projet de TCSP ne se limite pas à ses aspects techniques. C'est aussi un problème politique qui touche de nombreux aspects de la gouvernance. La politique de transport doit s'articuler avec d'autres orientations qui touchent, par exemple, au logement ou la localisation des pôles économiques. Les acteurs publics disposent pour cela d'un certain nombre d'outils de planification tels le Schéma d'Aménagement Régional (1998); le Plan de Déplacements Urbains de la CACEM (2003) ; le Schéma de Cohérence Territoriale (SCOT, en cours d'élaboration dans la CACEM) ; les POS et les Plans Locaux d'Urbanisme (PLU, en cours de réalisation); le Schéma de Mise en valeur de la Mer (pour la protection des espaces littoraux), etc. Or, ces documents de planification ne sont souvent que des compromis "parce qu'un consensus, même très vague, très général ou très lointain, n'existe que dans les discours officiels » (Martouzet, p.219). Les rivalités entre autorités institutionnelles ont longtemps favorisé l'immobilisme, contribuant ainsi à la dégradation des conditions de déplacement et à l'étalement de l'urbanisation sur le territoire martiniquais.

21 Seul un volontarisme politique fort est capable d'apporter des solutions réellement efficaces à cette situation. Par exemple, il a fallu de longues négociations avec les chauffeurs de taxicos interurbains (environ 400 de nos jours) pour réorganiser une profession dominée par de très petites entreprises, rassemblées en groupements d'intérêt économique (GIE). Des conventions passées entre ces GIE et le Conseil Général, aux risques et périls des transporteurs, sont censées fixer les modalités d'exploitation des services (horaires, tarifs, etc.) mais dans la réalité, la profession est confrontée à des difficultés financières qui se répercutent sur la qualité de l'offre de services. Pour défendre leurs intérêts, les transporteurs n'hésitent pas à avoir recours à la menace des barrages routiers qui paralyseraient ainsi l'île tout entière. En 2009, un syndicat de taxis collectifs a même saisi le tribunal administratif de Fort-de-France contre le Conseil Général pour demander l'annulation de la délibération relative à l'organisation du TCSP, demande rejetée par le rapporteur public.

Un autre aspect fondamental de cette politique publique est la politique de tarification retenue, elle-même associée à la gestion financière du projet. Il s'agit de trouver un niveau de prix à la fois acceptable par le client et permettant de dégager des excédents d'exploitation afin d'alléger le coût du projet pour les collectivités. Pour l'heure, la tarification envisagée prévoit un ticket unique pour la correspondance entre le TCSP et les autobus de la CACEM, et une politique sociale de $20 \%$. Les utilisateurs des parcsrelais paieront un léger supplément. Cette politique tarifaire est essentielle si l'on veut promouvoir le report modal auprès des usagers de la voiture.

\section{Les limites du projet de TCSP en matière de transport et de mobilités}

23 Le TCSP peut-il réellement apporter une solution efficace aux difficultés de déplacements à la Martinique? Rien n'est moins sûr et la révision «à la baisse » du projet risque fort d'en limiter les effets pour de nombreuses raisons.

24 Tout d'abord, on peut s'interroger sur la faible capacité des parcs-relais de Mahault (130 places) et de Carrère (250 places dans un premier temps, puis 500 places dans un 
second temps) compte tenu des contraintes de l'emprise foncière de ces équipements. Cela suffira-t-il pour inciter les automobilistes à y laisser leur véhicule pour emprunter le TCSP ? D'autres expériences de ce type ont déjà été menées comme par exemple l'ouverture, en 2009, d'une ligne de bus à Fort-de-France entre le parking de Dillon et le centre-ville (bibliothèque Schœlcher) afin de contribuer, même modestement, à solutionner les difficultés de stationnement dans l'hyper-centre. Pour de ne pas toucher uniquement la clientèle captive, l'offre de services de transport en commun doit devenir suffisamment attractive en termes de connexions, de fréquences, d'horaires et de fiabilité, pour arriver à détourner les automobilistes de leur véhicule. Or, l'attachement du Martiniquais à sa voiture est un phénomène reconnu, et on pourrait même dire «culturel »... L'élévation des niveaux de vie, le recours généralisé au crédit, la multiplication des mobilités, la reconnaissance sociale associée à la voiture ont ancré durablement la Martinique dans l'ère du tout-automobile. De plus, la dispersion de l'habitat, le mitage du territoire, rendent difficile la desserte par un mode collectif. Outre ses performances techniques, et au-delà d'obéir à un quelconque " effet de mode", le choix initial du tramway avait été réalisé pour apporter une image suffisamment forte, innovante et moderne afin de séduire le plus grand nombre. On peut craindre que le BHNS n'ait pas le même impact sur les populations...

Enfin, il ne faudrait pas focaliser les efforts uniquement sur une portion de l'agglomération foyalaise, en tentant de résorber le principal «point noir» de la circulation de l'île. Cela risque de déplacer partiellement les problèmes de congestion en amont des gares multimodales, sans apporter non plus de réelle solution à l'étalement urbain. Les impacts des infrastructures dépendent donc pour beaucoup des mesures d'accompagnement destinées à valoriser les équipements et de la volonté des populations à modifier leurs habitudes de mobilité au profit de modes de transport plus sûrs et plus respectueux de l'environnement.

\section{De nombreux autres enjeux déterminants}

Le TCSP pourrait également avoir des impacts sur les dynamiques urbaines ainsi que sur la situation socio-économique et environnementale des territoires.

\section{A. L'impact sur les recompositions urbaines}

Les mobilités et les systèmes de transport façonnent les territoires urbains et contribuent à leurs dynamiques. «Le tramway favorise aussi l'émergence de polarités secondaires autour de la multimodalité » [Laisney (dir.), Grillet-Aubert, 2006, p.138]. À l'échelle de l'agglomération foyalaise, le projet de TCSP ambitionne ainsi de faire émerger localement des pôles attractifs et multimodaux qui pourraient naître autour des terminus du TCSP, transformés en pôles d'échanges accueillant gares routières et parcs-relais. Leur fonction de concentration et de redistribution des flux en provenance du sud (Carrère), de l'est (Mahault) ou du nord (Pointe Simon) de l'île, peut être l'occasion de valoriser une situation de carrefour. À l'échelle intra-urbaine, le transport public est un outil pour redonner de la qualité à l'espace public et relancer l'attractivité des centres (Beaucire, 1996). À Fort-de-France, le TCSP est par exemple intégré dans l'aménagement du front de mer qui ouvre la ville vers la mer (construction d'un parvis piéton regroupant plusieurs pôles) et concourt à créer de l'animation, notamment dans 
un centre-ville déserté dès la nuit tombée. Il favorise une requalification urbaine de certains espaces, tels l'avenue Maurice Bishop et les quartiers attenants, peu attractifs aujourd'hui en termes d'image et de qualité du cadre de vie. Pour la municipalité de Fort-de-France, le projet permet d'envisager un élargissement de cette avenue prévu depuis longtemps dans le POS. Le site propre donne l'opportunité de redessiner les caractéristiques des voiries, de réaménager l'espace public au profit des piétons et des deux-roues.

Pour être pleinement efficace en matière d'aménagement du territoire, le projet de TCSP a tout intérêt à être prolongé vers les communes voisines, favorisant ainsi le développement de pôles urbains secondaires qui permettraient de contrebalancer le poids de l'agglomération foyalaise. L'organisation du transport pourrait se faire autour des bourgs existants qu'il faudrait promouvoir sous la forme de villes organisées en réseau. L'installation de gares dans ces pôles fonctionnerait comme des leviers au développement commercial et résidentiel. Mais toute politique volontariste en matière de transports doit s'accompagner d'autres mesures, en particulier celles portant sur l'utilisation des sols. Certains experts (Région, SETEC, 2001) recommandent une densification du bâti ainsi qu'une diversification des activités économiques autour des gares multimodales afin d'assurer un renforcement de l'armature urbaine, en accord avec les orientations des différents documents d'aménagement. Dans cette optique, les espaces intermédiaires (entre les bourgs) feraient l'objet d'une protection vis-à-vis de la construction afin de limiter les problèmes de spéculation ou d'endiguer le phénomène d'étalement urbain.

\section{B. Les enjeux économiques et sociaux}

Le TCSP n'est pas seulement un outil de désenclavement, il peut également devenir un levier du développement économique et social. En effet, selon la théorie des effets structurants, les infrastructures de transport sont une condition nécessaire, bien que non suffisante, à la croissance économique. Ce lien de causalité directe est cependant démenti dans de nombreux cas qui tendent à remettre en cause cette théorie (Offner, 1993). La rentabilité socio-économique du TCSP peut pourtant se mesurer notamment par les gains de temps et les effets induits procurés par l'utilisation de l'infrastructure. Actuellement, les embouteillages quotidiens rallongent les distances-temps et ont des conséquences sur la compétitivité des entreprises : retards dans les livraisons, perte de productivité des salariés... Le TSCP dessert sur son parcours des équipements qui drainent des flux intenses : les principaux pôles commerciaux (le centre-ville de Fortde-France et les centres commerciaux de Dillon et de la Galleria), les zones industrielles et artisanales situées le long de l'autoroute au Lamentin, plusieurs établissements scolaires et les principaux équipements culturels du centre-ville. Il peut contribuer à améliorer l'image de l'île auprès de la clientèle touristique, peu encline à se retrouver bloquée dans des embouteillages durant ses vacances, et met en relation directe l'aéroport international (qui n'est actuellement desservi par aucun transport en commun), la gare maritime inter-îles et le terminal de la Pointe Simon.

En plus d'améliorer les dessertes, ce type d'infrastructure peut être à l'origine d'un nouveau dynamisme du marché foncier, immobilier et commercial. L'une des difficultés à Fort-de-France se pose le long de l'avenue Maurice Bishop (section 2) où les travaux d'élargissement de la voie nécessitent une expropriation et un relogement des 
habitants concernés. La requalification urbaine de cet axe (alignement des bâtiments, etc.) pourrait valoriser le prix du foncier et de l'immobilier dans les quartiers populaires environnants, permettant ainsi une plus grande mixité sociale. Pour l'heure, le TCSP n'a semble-t-il pas été à l'origine d'« effets d'annonce » qui se soient, jusqu'ici, réellement concrétisés. Des projets de création de zones d'activités économiques existent sur le territoire de la CACEM, mais à moyen et à long termes : Étang Z’Abricots et La Trompeuse à Fort-de-France, Mangot Vulcin au Lamentin, etc. La réalisation du TCSP pourrait bien renforcer le pouvoir d'attraction de l'agglomération, ce qui ne va pas dans le sens d'un desserrement des activités économiques vers les communes voisines. Cet équilibre difficile à trouver illustre ainsi les contraintes pour un aménagement cohérent du territoire martiniquais. Si le projet est mené dans sa globalité, il sera mieux à même d'offrir aux populations une plus grande égalité des chances de se déplacer en tout lieu. L'impact sera nécessairement bien plus modeste si l'on se contente de se focaliser sur un axe restreint et sans réelle cohérence avec les autres modes de transport.

\section{Des impacts environnementaux mieux pris en compte}

31 L'espace martiniquais est soumis à de nombreuses contraintes telles l'insularité, l'exiguïté du territoire, un relief accidenté qui entraîne une concentration des hommes et des activités, des risques naturels prégnants, des écosystèmes riches mais fragiles, etc. Or, il est bien connu que l'accroissement des trafics et des réseaux de transport contribue à engendrer des nuisances pour l'homme et l'environnement (Bavoux et al., 2005). À l'échelle nationale, alors que l'État avait renoncé, en 2003, à subventionner les projets de TCSP, il a depuis affirmé son engagement en faveur d'une mobilité urbaine durable dans le cadre de la loi Grenelle de l'environnement $n^{\circ} 2$ du 12 juillet 2010 . La mise en place des TCSP, en réduisant le nombre de voitures en circulation, est avancée comme un des moyens pour lutter contre les nombreuses nuisances générées par les déplacements en véhicules à moteur: pollution de l'air, bruit, pollution des milieux aquatiques, etc.

La loi sur l'air et l'utilisation rationnelle de l'énergie (LAURE) de 1996 impose à toutes les agglomérations de plus de 100000 habitants une surveillance de la qualité de l'air. Celle-ci est assurée en Martinique par l'association Madininair, créée en 1998. Les informations recueillies, par la méthode des tubes passifs, au sein de l'agglomération foyalaise permettent de mesurer et de localiser les espaces les plus touchés par la pollution automobile. Les principaux polluants émis par le trafic routier sont le dioxyde d'azote $\left(\mathrm{NO}_{2}\right)$, l'oxyde d'azote (NO), les particules en suspension, etc. Les deux premiers sont des gaz irritants pour les bronches qui entraînent une hyper réactivité bronchitique chez les asthmatiques et qui favorisent les infections pulmonaires chez les enfants. Les mesures réalisées montrent que la pollution atmosphérique générée par le $\mathrm{NO}_{2}$ dans l'agglomération foyalaise se concentre le long des axes routiers principaux : la $\mathrm{N} 2$, la rocade (surtout au niveau du tunnel), l'autoroute (zone industrielle de la Jambette) et l'avenue Maurice Bishop. Les valeurs moyennes enregistrées sont ici bien supérieures aux normes limites annuelles pour la protection de la santé fixées à 40 microgrammes par mètre cube ${ }^{9}$. Le trafic automobile est également source de pollution sonore par l'intermédiaire du bruit des moteurs et d'échappement, les klaxons, etc. La baisse prévisible des trafics et la réduction des vitesses devraient permettre de réduire les nuisances sonores à proximité du tracé du TCSP. 
Malgré les différentes réglementations concernant la protection des milieux aquatiques (loi sur l'eau de 1992, loi sur l'eau et les milieux aquatiques de 2006) qui obligent les maitres d'ouvrages à collecter et à traiter les eaux pluviales qui ruissellent sur les sols imperméabilisés, de nombreux polluants (métaux lourds, etc.) provenant des véhicules peuvent se retrouver, par ruissellement, dans les cours d'eau, puis dans le milieu marin. D'après diverses études (SMTCSP, SYSTRA, 2006), la réalisation de certains tronçons du TCSP et de nouveaux ouvrages aura un impact sur l'écoulement des eaux en cas de crue. En effet, le risque d'inondation est fort sur cet itinéraire, en particulier au niveau de la rivière Lézarde, entre le Canal du Lamentin et l'aéroport. De plus, les travaux empiètent sur une zone de mangrove autour de l'échangeur du Canal du Lamentin. La construction de certaines infrastructures associées au projet (atelier dépôt et gare de Carrère) est prévue sur des zones non encore urbanisées. Compte tenu de tous ces éléments, le projet de TCSP ne pourra qu'être un compromis entre les données économiques, techniques et environnementales.

\section{Conclusion}

Les difficultés croissantes de déplacement à la Martinique, et en particulier dans l'agglomération foyalaise, sont révélatrices des déséquilibres territoriaux dans un espace insulaire fortement contraint et dans lequel l'hégémonie de la voiture particulière n'a jusqu'ici trouvé aucune parade, faute d'un engagement suffisamment fort de la part des acteurs politiques et des usagers. Le projet de TCSP et les blocages auquel il doit faire face illustrent les difficultés pour résoudre une situation dont la prise de conscience est réelle, mais jusqu'ici peu suivie d'effets. Le véritable enjeu est d'arriver à changer les mentalités pour inciter les populations à se tourner davantage vers des modes de transports durables (covoiturage, déplacements doux et transports collectifs). Mais pour cela, il faut modifier l'image des transports publics, en mettant en place une offre attractive, régulière et intermodale.

Le TCSP tant attendu ne permettra certainement pas de résoudre l'ensemble des dysfonctionnements en matière de circulation dans l'île, tout au plus évitera-t-il une dégradation à l'excès de la situation présente. Face à l'ampleur des enjeux, c'est tout un modèle de développement économique et de société qui est remis en cause.

\section{BIBLIOGRAPHIE}

Bavoux J.-J., Beaucire F., Chapelon L., Zembri P., 2005, Géographie des transports, Paris, Armand Colin, $232 \mathrm{p}$.

Beaucire F., 1996, Les transports publics et la ville, Toulouse, Éditions Milan, 63 p.

Brocard M. (dir.), 2009, Transports et territoires. Enjeux et débats, Paris, Ellipses, 184 p.

Communauté d'Agglomération du Centre de la Martinique, 2003, Le plan de déplacement urbain de la CACEM, $106 \mathrm{p}$. 
Conseil Régional de la Martinique, SETEC Économie, 2001, Étude multimodale de déplacements. Rapport de phase préliminaire, $66 \mathrm{p}$.

DREAL Midi-Pyrénées, 2012, Les trafics routiers sur le réseau national en région Midi-Pyrénées, Direction régionale de l'environnement, de l'aménagement et du logement, $4 \mathrm{p}$.

Gatineau L., Hoarau K., 2011, L'accessibilité à la « capitale » dans les espaces insulaires riches, une étude comparée Martinique/Réunion, Communication au colloque « Transports et territoires insulaires », Université des Antilles et de la Guyane, Campus de Schœlcher, 4-5 avril 2011.

GÉODE Caraïbe, 1998, Quels transports dans les petites îles caraibes ?, Paris, Karthala, coll. Terres d'Amérique, $\mathrm{n}^{\circ} 1,342 \mathrm{p}$.

Hamman P. (dir.), 2011, Le tramway dans la ville. Le projet urbain négocié à l'aune des déplacements, Rennes, Presses Universitaires de Rennes, 288 p.

INSEE Antilles-Guyane, 2010, Tableaux Économiques Régionaux, Martinique, 2009-2010, Pointe-à-Pitre, $171 \mathrm{p}$.

Laisney F., 2011, Atlas du tramway dans les villes françaises, Paris, Éditions Recherches, 424 p.

Laisney F. (dir.), Grillet-Aubert A., 2006, Tramway, espaces publics et mobilités, Architecture du transport, rapport final, vol. 3-A, Paris, IPRAUS-EAPB (Institut Parisien de Recherche :

Architecture, Urbanistique, Sociétés - École d'Architecture de Paris Belleville), 301 p.

Lévy J.-P., Dureau F., 2002, L'accès à la ville. Les mobilités spatiales en questions, Paris, L'Harmattan, $411 \mathrm{p}$.

Martouzet D., 2001, Fort-de-France, ville fragile?, Paris, Anthropos, 259 p.

Offner J.-M., 1993, « Les effets structurants du transport : mythe politique, mystification scientifique ", L’Espace Géographique, n³, p.233-244.

Offner J.-M., Pumain D., 1996, Réseaux et territoires, significations croisées, La Tour d'Aigues, Éditions de l'Aube, $280 \mathrm{p}$.

Pélis Y., Saffache P., Ranély Vergé-Dépré C., 2007, « Les facteurs à l'origine de la mise en place d'un Transport en Commun en Site Propre (TCSP) dans l'agglomération de Fort-de-France (Martinique) ", Études caribéennes, $n^{\circ} 8$.

Syndicat Mixte du TCSP de la Martinique, SYSTRA, 2006, Dossier d'enquête préalable à la déclaration d'utilité publique, 2 vol., 58 p. et 243 p.

\section{NOTES}

1. "Martinique, le risque d'asphyxie des transports ", L'Express, 12/06/2003.

2. "Trois projets de TCSP retenus en Outre-mer », France-Antilles Martinique, 10/02/2011. Outre celui de la Martinique, il s'agit de deux projets de BHNS à La Réunion.

3. De 1992 à 2011, le Conseil Général a été présidé par Claude Lise (fondateur d'un parti de gauche, le Rassemblement démocratique martiniquais) et de 1998 à 2010, le Conseil Régional avait à sa tête Alfred Marie-Jeanne, leader du Mouvement Indépendantiste Martiniquais.

4. Le Conseil Général envisageait un tracé passant par les quartiers densément peuplés de SainteThérèse et de Dillon, au sud de l'autoroute. L'autre tracé, soutenu par le Conseil Régional et la DDE, proposait un élargissement de l'autoroute pour le passage du TCSP.

5. En 2011, 28 villes françaises sont équipées d'un tramway (Laisney, 2011).

6. «Chauvet: Claude Lise perd les pédales du TCSP », Politiques Publiques, 15/03/2011. 
7. «Le TCSP a failli dérailler ", France-Antilles Martinique,08/06/2011.

8. Le 24 janvier 2010, les Martiniquais votèrent pour la création d'une COM dans le cadre de l'article 73 de la constitution.

9. Madininair, Rapport d'activités 2011, Fort-de-France, 44 p.

\section{RÉSUMÉS}

L'agglomération de Fort-de-France est confrontée à de graves problèmes de circulation routière qui sont révélateurs de son rôle polarisateur sur l'île de la Martinique tout entière. Cette situation impose donc une politique des transports cohérente dans le cadre de projets d'urbanisme et d'aménagement du territoire. Il s'agit de lutter contre la toute puissance de l'automobile en proposant une offre alternative basée sur l'amélioration des transports collectifs grâce à la mise en place d'un TCSP.

Alors que la réflexion a été engagée depuis une quinzaine d'années, la réalisation encore en cours du TCSP rencontre des difficultés de natures diverses. Projet ambitieux de tramway ou simple voie réservée aux autobus, le futur TCSP de la Martinique peut-il répondre aux différentes attentes des élus, des aménageurs ou à celles des populations qui subissent les embouteillages au quotidien?

The urban area of Fort-de-France is confronted with serious problems of road traffic which emphasize the city's centralizing role on the island of Martinique. This situation thus imposes a coherent transport policy in the context of urban planning and country planning. To fight against the overwhelming power of cars proposals have been made for an alternative system based on the improvement of public transport thanks to establishment of a « TCSP ».

While the project of «TCSP» has caused brainstorming for about fifteen years, its implementation still encounters various difficulties. Whether it is an ambitious tram project or a simple bus lane, can the future «TCSP » of Martinique meet the different expectations of elected representatives, planners and users who have to endure traffic jams on a daily basis?

\section{INDEX}

Keywords : accessibility, urban area of Fort-de-France, Martinique, TCSP, tram

Mots-clés : accessibilité, agglomération foyalaise, Martinique, TCSP, tramway

\section{AUTEUR}

\section{COLETTE RANÉLY VERGÉ-DÉPRÉ}

Maître de Conférences, AIHP-GÉODE Caraïbe (EA 929).Université des Antilles et de la Guyane. colette.ranely[chez]iufm-martinique.fr 